\title{
MODELAGEM E SOLUÇÃO DE UM PROBLEMA DE TRANSPORTE DE CONTAMINANTES UTILIZANDO O MÉTODO DOS VOLUMES FINITOS
}

\author{
Anna Luisa Soares Castro Coimbra \\ Instituto do Noroeste Fluminense de Educação Superior (INFES), Universidade \\ Federal Fluminense (UFF) \\ Av. João Jasbick, s/n, Aeroporto, Santo Antônio de Pádua, RJ, Brasil \\ anna_luisa@id.uff.br \\ Wagner Rambaldi Telles \\ Instituto do Noroeste Fluminense de Educação Superior (INFES), Universidade \\ Federal Fluminense (UFF) \\ Av. João Jasbick, s/n, Aeroporto, Santo Antônio de Pádua, RJ, Brasil \\ wtelles@id.uff.br
}

\section{RESUMO}

O controle do comportamento de contaminantes em rios é extremamente útil para processos de tomada de decisão, por exemplo, para determinar o melhor local para descarte de resíduos domésticos e industriais, evitando assim uma contaminação do meio ambiente e contribuindo para um melhor monitoramento da qualidade da água, sendo que até mesmo a Organização das Nações Unidas enfatiza a importância de se ter um exímio controle sobre a qualidade das águas nos rios. Neste trabalho foi utilizado o Método dos Volumes Finitos com formulação implícita, o qual tem como pilar as integrais múltiplas e as séries de Taylor, aplicado na solução numérica de um modelo matemático unidimensional, comparando os resultados com dados experimentais retirados da literatura. Foi observado que os valores numéricos se aproximam do problema real relacionado à dispersão de contaminantes em rios.

Palavra-chave: Poluição Hídrica; Transporte de Contaminantes; Modelagem Computacional; Método dos Volumes Finitos.

\section{ABSTRACT}

Control of contaminant behavior in rivers is extremely useful for decision-making processes, for example to determine the best disposal site for domestic and industrial waste, thus avoiding environmental contamination and contributing to better monitoring of water quality. Even the United Nations emphasizes the importance of excellent control over water quality in rivers. In this work we used the Finite Volume Method with implicit formulation, which is based on multiple integrals and Taylor series, applied in the numerical solution of a one-dimensional mathematical model, comparing the results with experimental data 
from the literature. It was observed that the numerical values approach the real problem related to the dispersion of contaminants in rivers.

Keywords: Water Pollution; Contaminant Transport; Modeling Computational; Finite Volume Method.

\section{Como Citar:}

COIMBRA, A. L. S. C.; TELLES, W. R. Modelagem e Solução de um Problema de Transporte de Contaminantes Utilizando o Método dos Volumes Finitos. In: SIMPÓSIO DE PESQUISA OPERACIONAL E LOGÍSTICA DA MARINHA, 19., 2019, Rio de Janeiro, RJ. Anais [... ]. Rio de Janeiro: Centro de Análises de Sistemas Navais, 2019.

\section{INTRODUÇÃO}

Atualmente se vive em um dos piores momentos possíveis relacionado ao conceito de poluição hídrica, em especial, dos rios. O cenário é preocupante devido ao fato de essa poluição ser uma das causadoras da escassez de água potável.

Neste contexto, a poluição é resultado indesejável das ações de transformação das características naturais de um ambiente, atribuindo um caráter nocivo a qualquer utilização que se faça do mesmo (Bastos; Freitas, 2006, p. 39). Cabe ressaltar ainda, que boa parte da água potável proveniente dos rios sofre constante ameaça de poluição, devido à expansão urbana, aliada ao desenvolvimento da indústria e das atividades agrícolas.

A Organização das Nações Unidas enfatiza a necessidade de uma maior fiscalização no que se refere à qualidade da água nos rios, mas na prática é muito diferente, visto que o monitoramento e avaliação pelos órgãos responsáveis acabam acarretando problemas financeiros e logísticos, já que se necessita de material específico e mão de obra qualificada para o trabalho, tornando assim o processo caro.

Nesse contexto, torna-se essencial a utilização de modelos matemáticos - e consequente solução numérica envolvendo algoritmos computacionais - para simular o transporte e dispersão de poluentes em cursos d'água naturais, uma vez que estes podem contribuir para o processo de tomada de decisões, possibilitando a geração de diferentes cenários realísticos sem a necessidade de vários trabalhos de campo para essa finalidade, agregando um melhor custo/benefício no que tange a análise desse tipo de problema.

Diante do exposto, com o intuito de realizar a calibração e validação de um modelo de qualidade de água, foi analisado o lançamento de um traçador salino em um trecho do rio São Pedro, pequeno curso d'água situado na bacia hidrográfica do rio Macaé, centro-norte do Estado do Rio de Janeiro. Para isso, utilizou-se os dados do trabalho de campo realizado por Sousa (2009). A partir dos dados obtidos no campo, foi realizada a modelagem do problema a fim de obter a equação (modelo matemático) mais representativa possível, a qual foi resolvida pelo Método dos Volumes Finitos (MVF), método esse que vem sendo utilizado nas mais diversas áreas da ciência, cujo objetivo foi reproduzir numericamente o comportamento da concentração salina em um ponto de interesse do referido rio e comparar esses resultados com os dados experimentais correspondentes. 


\section{ESTUDO DE CASO}

\section{1. Área de Estudo - Rio São Pedro}

A área de estudo localiza-se na Região Serrana do Estado do Rio de Janeiro dentro da bacia do rio Macaé. Dentre as bacias hidrográficas localizadas unicamente no Estado, essa é a de maior extensão, com uma área de drenagem de aproximadamente $1.765 \mathrm{~km}^{2} \mathrm{e}$ com população em torno de 141.000 habitantes. Essa bacia engloba praticamente toda a área dos limites territoriais do município de Macaé, com cerca de $1.448 \mathrm{~km}^{2}$, e ainda áreas dos municípios de Nova Friburgo $\left(142 \mathrm{~km}^{2}\right)$, Casimiro de Abreu $\left(83 \mathrm{~km}^{2}\right)$, Rio das Ostras $\left(11 \mathrm{~km}^{2}\right)$, Conceição de Macabu $\left(70 \mathrm{~km}^{2}\right)$ e Carapebus $\left(11 \mathrm{~km}^{2}\right)$ (Sousa, 2009).

Nos últimos anos o rio Macaé vem sendo deteriorado, seja na qualidade da água ou na destruição das matas ciliares. Como consequência destas intervenções destacam-se o assoreamento do rio e restrições ao uso da água para o consumo (Costa, 1999).

Segundo Telles (2009), intensas atividades petrolíferas desenvolvidas na região estuarina vêm causando grandes impactos ambientais. Em consequência disso, áreas de grande potencial de riquezas naturais e inúmeras unidades biológicas vem sendo afetadas, acarretando danos irreversíveis ao meio ambiente e aos ecossistemas presentes na região.

\subsection{Descrição do Experimento}

A região escolhida para a realização do experimento está situada em São Pedro da Serra, $7^{\circ}$ Distrito de Nova Friburgo-RJ, local que foi afetado por um rápido processo de transformação espacial, social e econômica, com fortes impactos ambientais (LimaVerde, 2005). Na Figura 1 é mostrada a região de interesse.

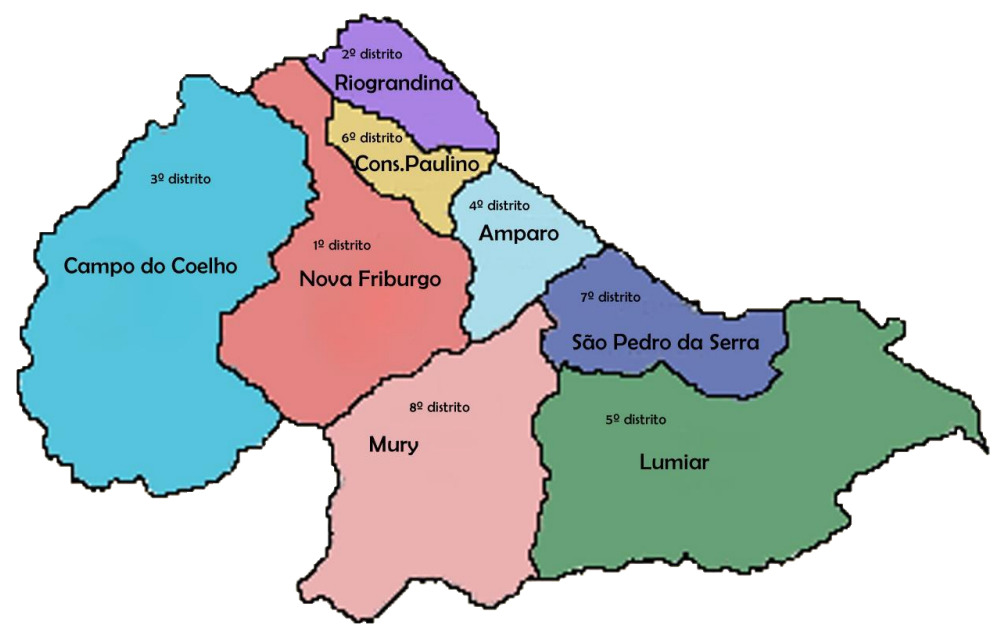

Figura 1: Localização do distrito de São Pedro da Serra. Fonte: Ribeiro (2016).

O experimento descrito no trabalho de Sousa (2009), o qual foi base para essa pesquisa, ocorreu no dia 26 de janeiro de 2009, com início às 10:50:28 h, e buscou simular um cenário de poluição acidental no curso de água em questão. Para isso, foi utilizada uma solucão de cloreto de sódio $(\mathrm{NaCl})$ como tracador. Se comparado com outros tracadores. 
o $\mathrm{NaCl}$ apresenta vantagens como, por exemplo, solubilidade em água, presença natural quase nula, não possui efeitos nocivos ao impacto ambiental, facilidade de armazenamento ou de quantificação e também um custo muito baixo. As medições foram feitas em um trecho do rio localizado na saída da vila, em frente à estrada do cemitério (22 19.155S, $4219.897 \mathrm{~W})$. Na Figura 2 é apresentada a vista parcial de interesse do trecho do rio São Pedro.

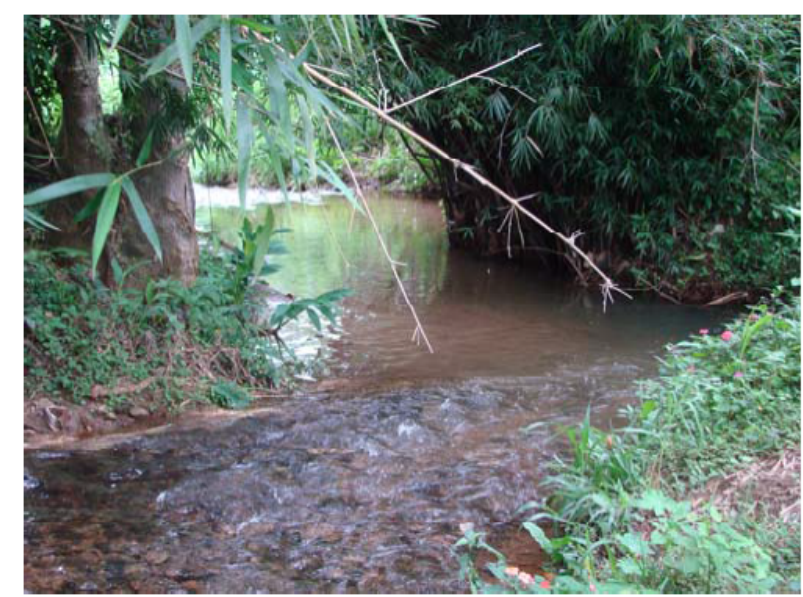

Figura 2: Vista parcial do trecho do rio São Pedro. Fonte: Sousa (2009).

Para a preparação da solução salina, foram utilizados $2.000 \mathrm{~g}$ de cloreto de sódio, diluídos em aproximadamente $15 l$ de água em um balde, os quais foram liberados instantaneamente em um ponto da seção de injeção, sobre a linha de corrente central do escoamento. A primeira seção foi escolhida a $50 \mathrm{~m}$ do ponto de injeção e a segunda a $100 \mathrm{~m}$. Foram colhidas amostras de $200 \mathrm{ml}$ da água a cada $15 \mathrm{~s}$, totalizando 60 amostras, as quais, posteriormente, foram analisadas em laboratório. Na Tabela 1 são mostradas as configurações do rio.

Tabela 1: Características do rio no ponto de lançamento do traçador.

\begin{tabular}{l|l}
\hline Parâmetros & Valores \\
\hline \hline Vazão $(Q=U A)$ & $0,43 \mathrm{~m}^{3} / \mathrm{s}$ \\
\hline Área $(A)$ & $1,05 \mathrm{~m}^{2}$ \\
\hline Largura $(B)$ & $2,40 \mathrm{~m}$ \\
\hline Profundidade $(H=A / B)$ & $0,43 \mathrm{~m}$ \\
\hline Velocidade $(U)$ & $0,41 \mathrm{~m} / \mathrm{s}$ \\
\hline Declividade $(S)$ & $0,03 \mathrm{~m} / \mathrm{m}$ \\
\hline Concentração inicial $\left(C_{0}\right)$ & $15,50 \mathrm{mg} / \mathrm{l}$ \\
\hline
\end{tabular}

\section{MODELO MATEMÁTICO}

O modelo matemático para o problema proposto neste trabalho leva em consideração o fato do trecho do rio estudado ser suficientemente estreito e raso, fazendo com que 
as variações nas direções transversal e vertical sejam desprezíveis em relação às variações longitudinais. Assim, um modelo unidimensional da Equação de Advecção-Dispersão, com condições inicial e de contorno adequadas, que leve em consideração apenas as variações na direção longitudinal pode ser empregado, sendo expresso da seguinte forma:

- Equação de Advecção-Dispersão:

$$
\frac{\partial C}{\partial t}+U \frac{\partial C}{\partial x}=E_{L} \frac{\partial^{2} C}{\partial x^{2}},-\infty<x<\infty, t>0 .
$$

- Condições inicial e de contorno:

$$
\begin{gathered}
C(x, 0)=C_{0}+\frac{M}{A} \delta(x), \quad-\infty<x<\infty \\
C( \pm \infty, t)=C_{0}, \quad t>0,
\end{gathered}
$$

onde $C$ é a concentração $(m g / l), t$ é a variável temporal $(s), x$ é a variável espacial $(m), U$ é a velocidade no sentido longitudinal do escoamento $(\mathrm{m} / \mathrm{s}), E_{L}$ é o coeficiente de dispersão longitudinal $\left(\mathrm{m}^{2} / \mathrm{s}\right), M$ é a massa do constituinte $(\mathrm{mg}), A$ é a área da seção transversal do rio $\left(m^{2}\right), \delta(x)$ é a função Delta de Dirac, que fisicamente representa uma unidade de massa de constituinte dentro de um espaço infinitamente pequeno e que tem a propriedade de ser nula no tempo inicial em todos os lugares, exceto em $x=0$ (posição de injeção de massa $M$ do constituinte) tal que:

$$
\int_{-\infty}^{\infty} \delta(x) d x=1 .
$$

A solução analítica para o modelo matemático descrito na equação (1), com condições de contorno e inicial, dadas pelas equações (2a) e (2b), é dada pela equação (4) (Sousa, 2009):

$$
C(x, t)=C_{0}+\frac{M}{A \sqrt{4 \pi E_{L} t}} \exp \left(-\frac{(x-U t)^{2}}{4 E_{L} t}\right),-\infty<x<\infty, t>0 .
$$

Já a solução numérica do problema aqui descrito, foi obtida empregado o Método dos Volumes Finitos com formulação implícita, o qual é descrito na próxima seção.

\subsection{Método dos Volumes Finitos}

O Método dos Volumes Finitos consiste em determinar um limite (domínio) para a região investigada. Em seguida, essa região é dividida em um número finito de volumes, denominados volumes de controle. Após esse processo, é feito o cálculo da integral múltipla da equação aplicada a cada volume, aproximando os valores das variáveis nas faces e as derivadas com a informação das variáveis nodais, obtendo um sistema algébrico ao final. A partir desse ponto, é construída uma matriz representando o sistema algébrico a ser implementado computacionalmente e resolvido numericamente utilizando um método apropriado, de acordo com a característica do problema. 
Para o trecho do rio São Pedro analisado neste trabalho foi realizada a discretização do domínio, dando origem às malhas espaciais, para que possa ser aplicado o Método dos Volumes Finitos, onde as letras $W$ e $E$ representam as direções Oeste e Leste, respectivamente, as letras $w$ e $e$ representam suas faces, por fim, a letra $P$ é centro do volume de controle. Na Figura 3 é apresentada uma representação ilustrativa da malha espacial unidimensional para o Método dos Volumes Finitos.

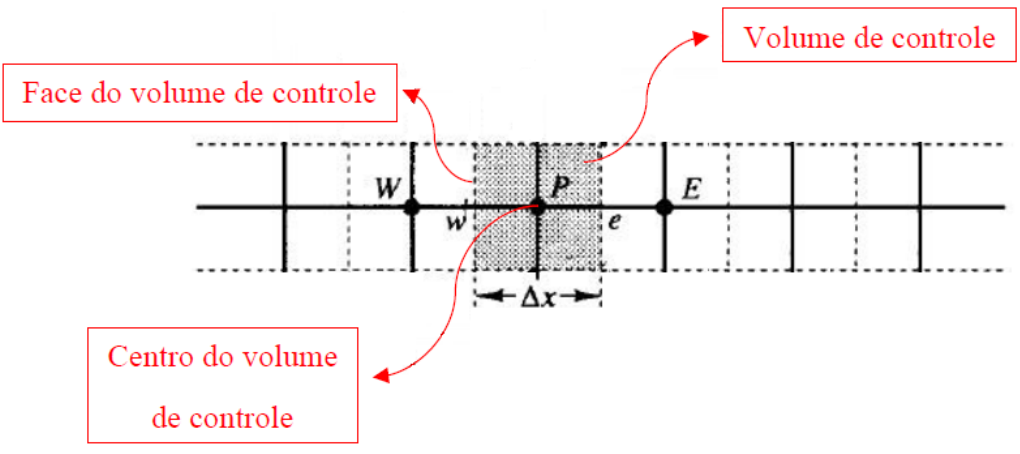

Figura 3: Ilustação da malha espacial para o Método dos Volumes Finitos. Fonte: Adaptado de Ferreira (2017).

Aplicando o método na equação (1), pode-se realizar a integração ao longo do tempo - em intervalos de tempo $\Delta \mathrm{t}$ (constantes) - e no espaço - de $w$ até $e$ com $\Delta x=e-w$ do termo transiente (primeiro termo do lado esquerdo da equação (1)) da referida equação, da seguinte forma:

$$
\int_{t}^{t+\Delta t} \int_{w}^{e} \frac{\partial C}{\partial t} d x d t=\Delta x\left(C_{P}^{t+\Delta t}-C_{P}^{t}\right)
$$

A integração do termo advectivo (segundo termo do lado esquerdo da equação (1)) pode ser feita através do esquema Upwind (UDS), o qual consiste na substituição do valor da função nas interfaces $w$ e $e$ de cada volume de controle pelo valor da mesma no ponto a montante, o qual varia de acordo com o sentido da velocidade do escoamento (Versteeg; Malalasekera, 1995), ou seja:

$$
\int_{t}^{t+\Delta t} \int_{w}^{e} U \frac{\partial C}{\partial x} d x d t=U \Delta t\left(C_{P}^{t+\Delta t}-C_{W}^{t+\Delta t}\right)
$$

Para a integração do termo dispersivo longitudinal (termo do lado direito da equação (1D) foi usada a expresão de Taylor para aproximar a derivada segunda em $x$, via diferenças centrais. Logo,

$$
\int_{t}^{t+\Delta t} \int_{w}^{e} E_{L} \frac{\partial^{2} C}{\partial x^{2}} d x d t=E_{L} \Delta t\left(\frac{C_{E}^{t+\Delta t}-2 C_{P}^{t+\Delta t}+C_{W}^{t+\Delta t}}{\Delta x}\right) .
$$

Substituindo as equações (5) a (7) na equação (1), obtém-se a seguinte formulacão para a Equacão de Adveccão-Dispersão unidimencional discretizada pelo Método dos 
Volumes Finitos com formulação implícita e condições de contorno e iniciais dadas pelas equações 2a e 2b:

$$
\begin{gathered}
\left(\frac{-U \Delta t \Delta x-E_{L} \Delta t}{\Delta x^{2}}\right) C_{W}^{t+\Delta t}+\left(\frac{\Delta x^{2}+U \Delta t \Delta x+2 E_{L} \Delta t}{\Delta x^{2}}\right) C_{P}^{t+\Delta t}+ \\
\left(\frac{-E_{L} \Delta t}{\Delta x^{2}}\right) C_{E}^{t+\Delta t}=C_{P}^{t}
\end{gathered}
$$

A equação (8) gera um sistema da forma $A \mathbf{x}=\mathbf{b}$, o qual foi resolvido numericamente pelo método Gauss-Seidel.

Para simular o lançamento instantâneo da solução salina, foi selecionada a célula que representasse, no modelo computacional, o ponto de lançamento adotado no domínio físico, assumindo-se imediata e completa homogeneização da solução salina em seu interior.

\section{RESULTADOS E DISCUSSÕES}

A fim de calibrar e validar o modelo matemático resolvido pelo Método dos Volumes Finitos, foram comparados os resultados numéricos obtidos com o mesmo com a solução analítica dada pela equação (4) e os dados experimentais obtidos no trabalho de campo descrito em Sousa (2009), cujos parâmetros são mostrados na Tabela 2 .

Os resultados apresentados neste trabalho foram gerados em um computador com processador Intel Core 2 Duo com 2 GB de memória RAM. Em todas as simulações realizadas, o custo computacional apresentou uma variação do tempo de processamento entre $30 s$ e $280 s$. Para a implementação computacional foi utilizado o software Scilab versão 5.5.0.

Tabela 2: Características da região de interesse no ponto de coleta das concentrações.

\begin{tabular}{l|l}
\hline Parâmetros & Valores \\
\hline \hline Concentração inicial do rio & $15,50 \mathrm{mg} / \mathrm{l}$ \\
\hline Largura média do rio no local do experimento & $2,40 \mathrm{~m}$ \\
\hline Profundidade média do rio no local do experimento & $0,43 \mathrm{~m}$ \\
\hline Comprimento do trecho do rio sob análise & $500 \mathrm{~m}$ \\
\hline Tempo do experimento (tempo final) & $550 \mathrm{~s}$ \\
\hline Posição de lançamento do poluente no domínio & $100 \mathrm{~m}$ \\
\hline Posição de coleta do poluente no domínio & $200 \mathrm{~m}$ \\
\hline
\end{tabular}

\subsection{Variação da Malha Espacial}

Primeiramente, foi realizada uma análise para a definição da melhor malha computacional a ser utilizada durante as simulações, levando em consideração o melhor custo e benefício em relação ao tempo computacional e ajuste dos resultados numéricos junto aos dados experimentais. 
Na Figura 4 são apresentados os perfis de concentração para a região de interesse com base nos parâmetros descritos na Tabela 2, e utilizando um número de volumes da malha espacial igual a 500 e o número de nós da malha temporal igual a 550, este último adotado também para as demais simulações. Já o valor do coeficiente de dispersão longitudinal foi de $1,82 \mathrm{~m}^{2} / \mathrm{s}$.

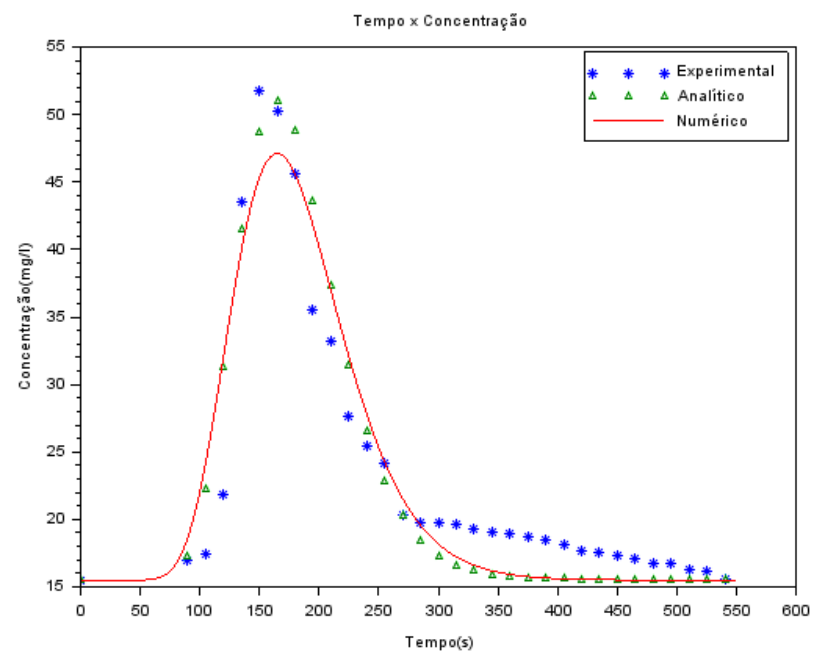

Figura 4: Comportamento da concentração no ponto de coleta com os parâmentros descritos na Tabela 2, com $\Delta x=1$ e $\Delta t=1$.

Posteriormente, com o intuito de verificar a influência da malha nos resultados obtidos, foi feita a variação da malha espacial buscando um melhor ajuste da solução numérica, como mostrado na Figura 5 , tomando como referência os parâmetros descritos na Tabela 2 e coeficiente de dispersão longitudinal igual a $1,82 \mathrm{~m}^{2} / \mathrm{s}$.

Foi possível observar que, a partir da malha com 700 volumes, os resultados pouco se diferenciam daqueles apresentados na malha com 500 volumes (Figura 4). Além disso, com 1.000 volumes na malha espacial o modelo tende a convergir, apresentando uma diferença mínima entre a Figura 5(c) (1.000 volumes) e a Figura 5(d), com 1.500 volumes. Por outro lado, o custo computacional para a implementação de 1.500 volumes foi de 280 $s$, mais que o dobro do tempo de execução com 1.000 volumes, o qual foi de $90 \mathrm{~s}$.

\subsection{Variação do Coeficiente de Dispersão Longitudinal}

Por fim, foram realizados ainda vários testes para determinar o coeficiente de dispersão longitudinal visando um melhor ajuste dos resultados numéricos frente à solução analítica e dados experimentais, parâmetro esse que, juntamente com a velocidade, é um dos mais críticos para a precisão das simulações geradas por esses modelos. No intuito de avaliar a variação desse coeficiente, utilizou-se os valores de $E_{L}=1,00 \mathrm{~m}^{2} / \mathrm{s}$ (Figura 6(a)), $E_{L}=1,83 \mathrm{~m}^{2} / \mathrm{s}$ (Figura 6(b)), $E_{L}=2,00 \mathrm{~m}^{2} / \mathrm{s}$ (Figura 6(c)) e $E_{L}=3,00 \mathrm{~m}^{2} / \mathrm{s}$ (Figura 6(d)). Os demais parâmetros necessários à simulação são os mesmos descritos na Tabela 2, tomando 500 nós para a malha espacial e 550 nós para a malha temporal.

Verifica-se que a melhor configuração do coeficiente de dispersão longitudinal foi o valor de $E_{T}=1.83 \mathrm{~m}^{2} / \mathrm{s}$. Figura 6(b), aue tem valor próximo ao encontrado por Sousa 


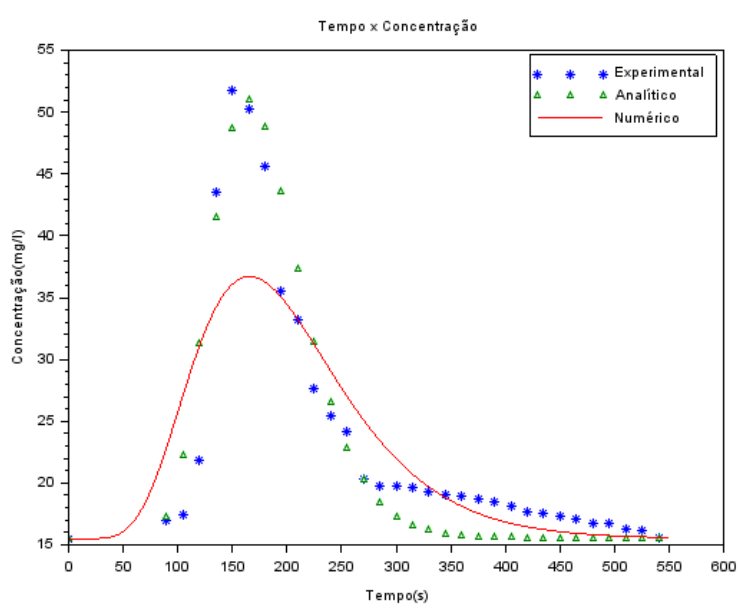

(a) 50 volumes na malha espacial.

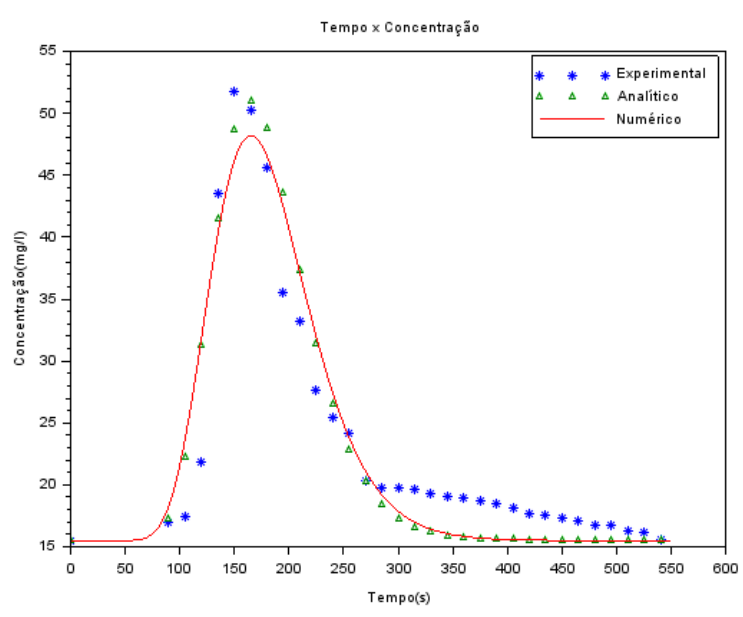

(c) 1.000 volumes na malha espacial.

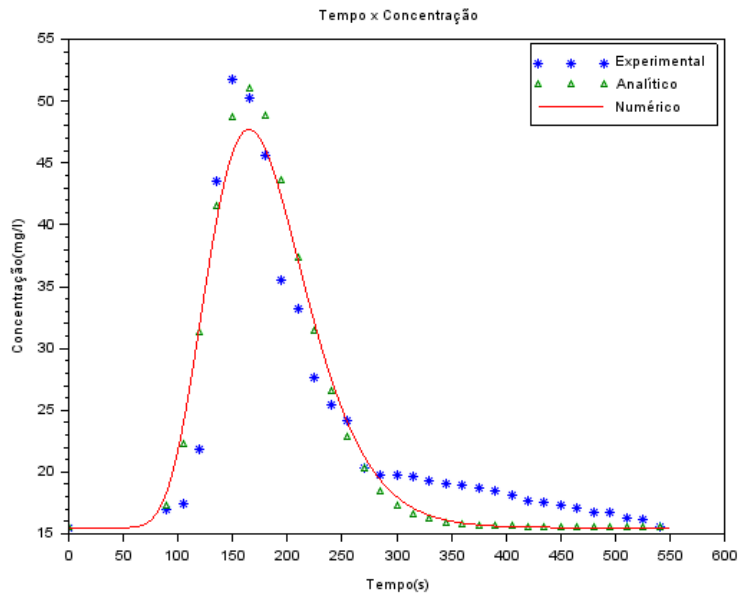

(b) 700 volumes na malha espacial.

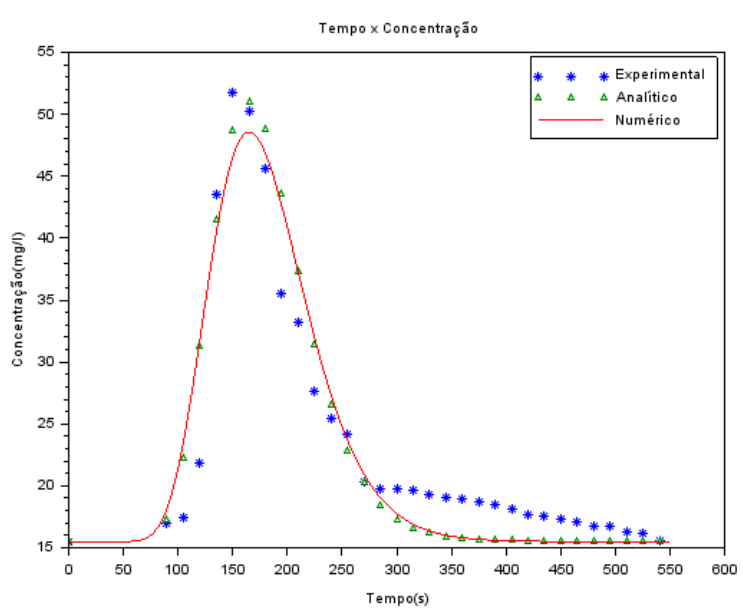

(d) 1.500 volumes na malha espacial.

Figura 5: Perfil das concentrações no ponto de coleta utilizando os parâmetros descritos na Tabela 2, com $\Delta t=1$ e diferentes discretizações para a malha espacial.

(2009), podendo-se observar que o resultado numérico está mais próximo ao analítico. 


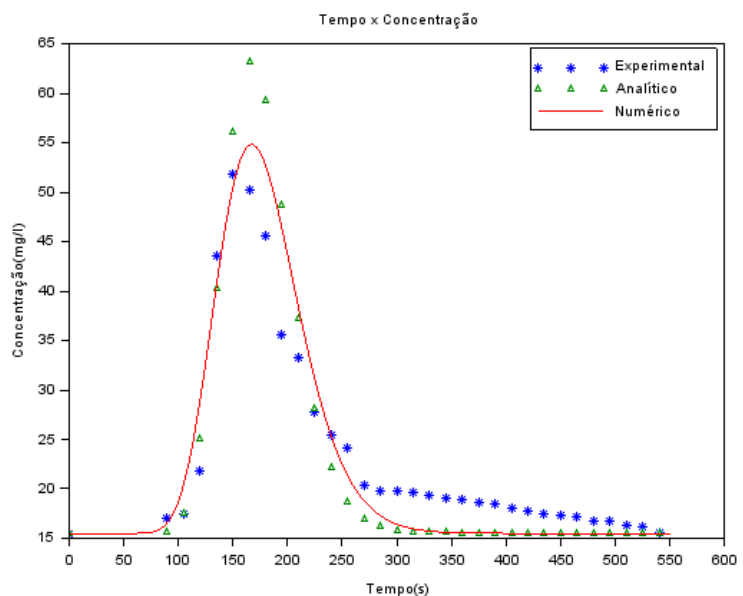

(a) $E_{L}=1,00 \mathrm{~m}^{2} / \mathrm{s}$.

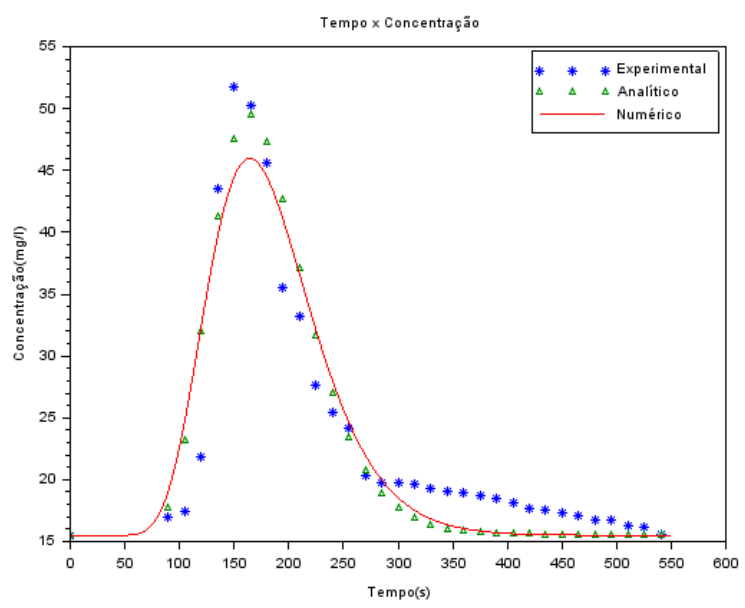

(c) $E_{L}=2,00 \mathrm{~m}^{2} / \mathrm{s}$.

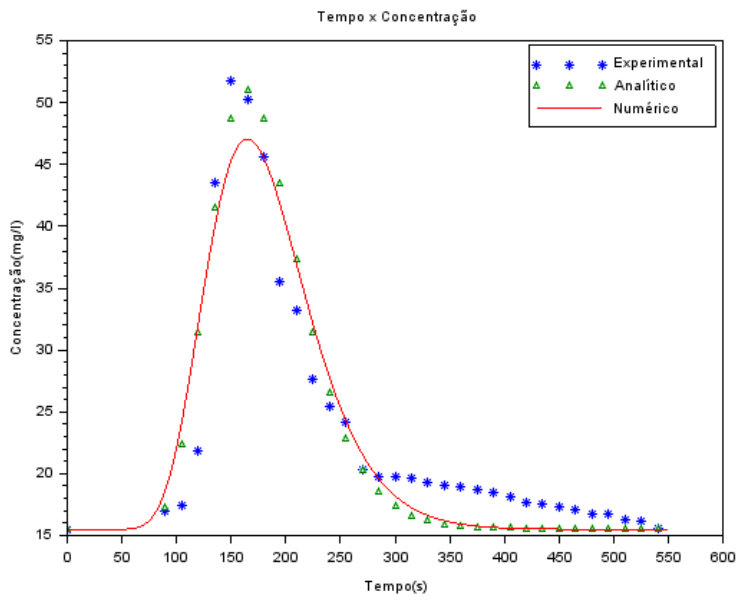

(b) $E_{L}=1,83 \mathrm{~m}^{2} / \mathrm{s}$.

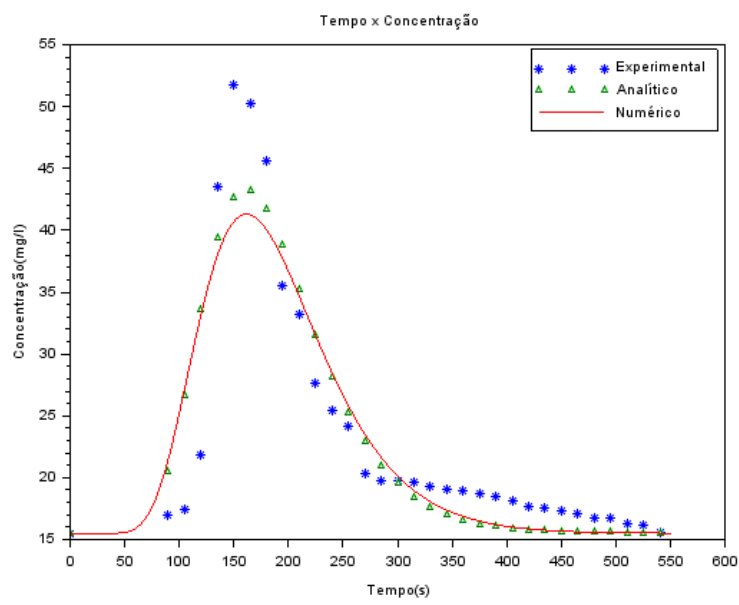

(d) $E_{L}=3,00 \mathrm{~m}^{2} / \mathrm{s}$

Figura 6: Perfil das concentrações no ponto de coleta utilizando diferentes valores para o coeficiente de dispersão longitudinal.

\section{CONCLUSÕES}

Diante das simulações realizadas e dos resultados apresentados na Seção 4, concluise, com a variação da malha espacial que, quanto maior a divisão da malha espacial, melhor será o resultado obtido. No entanto, esse refinamento acarreta em um maior tempo computacional necessário para realizar as simulações. Por outro lado, ao analisar a variação do coeficiente de dispersão longitudinal, verifica-se que o mesmo tem significativa influência na atenuação do pico de concentração da pluma de contaminante. Verifica-se também, uma pequena discrepância entre os resultados numéricos em relação a solução analítica e aos dados experimentais no que se refere ao pico de concentração, fato que carece de uma maior investigação futura.

Finalizando, constatou-se ainda que, após as variações dos parâmetros e comparação dos resultados numéricos, obtidos na resolução do modelo matemático utilizando o 
Método dos Volumes Finitos, com a solução analítica e os dados experimentais, foi possível calibrar o modelo para que as simulações por ele realizadas se comportassem de maneira satisfatória na região de interesse, o que motiva sua aplicação em corpos hídricos com características e geometrias similares.

Além disso, o modelo matemático aqui abordado, com solução numérica obtida por algoritmos computacionais para simular o transporte e dispersão de poluentes em cursos d'água naturais, pode contribuir para o processo de tomada de decisões na região de interesse, uma vez que permite a análise de diferentes cenários em menor tempo, sem a necessidade de gastos com trabalhos de campo.

\section{AGRADECIMENTOS}

Os autores deste trabalho agradecem à Universidade Federal Fluminense (UFF) e ao Conselho Nacional de Desenvolvimento Científico e Tecnológico (CNPq) pela bolsa de Iniciação Científica referente ao Programa Institucional de Bolsas de Inciação Científica (PIBIC).

\section{REFERÊNCIAS BIBLIOGRÁFICAS}

[1] BAStOS, A. C. S.; FREITAS, A. C. Agentes e processos de interferência, degradação e dano ambiental. In: CUNHA, S. B.; GUERRA, A. J. T. (Orgs.). Avaliação e Perícia ambiental. 7. ed. Rio de Janeiro: Bertrand Brasil, 2006.

[2] COSTA, H. Para gestão dos recursos hídricos das bacias hidrográficas dos rios Macacu, São João, Macaé e Macabu. Rio de Janeiro: Secretaria de Estado de Meio Ambiente e Desenvolvimento Sustentável (SEMADS), 1999.

[3] FERREIRA, T. R. N. O uso da Matemática na modelagem e solução de problemas reais em recursos hídricos. Trabalho de Conclusão de Curso (Graduação em Licenciatura em Matemática), Universidade Federal Fluminense, Instituto do Noroeste Fluminense de Educação Superior, 2017.

[4] LimaVerde, A. M. F.; AGUiAR, L. A. de; Villas V. B. S. R.; MELlO, J. F. S. Preservação dos mananciais do rio São Pedro: projeto interdisciplinar de educação ambiental. Revista Rio de Janeiro, n. 16-17, 2005.

[5] ONU. Organização das Nações Unidas. Declaração da "ONU Água" para o Dia Mundial da Água 2010, Nações Unidas no Brasil (ONUBR). Disponível em: $<$ https://nacoesunidas.org/acao/agua/>. Acesso em: 22 de dez. de 2016.

[6] SOUSA, E. P. Avaliação de Mecanismos Dispersivos em Rios Através de Problemas Inversos. Dissertação (Mestrado em Modelagem Computacional), Instituto Politécnico, Universidade do Estado do Rio de Janeiro, Nova Friburgo, RJ, 2009.

[7] RIBEIRO, M. A. C. O papel de Nova Friburgo na rede de localidades centrais fluminense: uma análise comparativa 1966-2007. Geo UERJ, Rio de Janeiro, n. 29, p. 452-472, 2016. 
[8] TELLES, W. R. Simulação do transporte horizontal bidimensional de substância conservativa. Dissertação (Mestrado em Modelagem Computacional), Instituto Politécnico, Universidade do Estado do Rio de Janeiro, Nova Friburgo, RJ, 2009.

[9] VERSTEEG, H. K.; MALALASEKERA W. An introduction to Computational Fluid Dynamics: The Finite Volume Method. Longman Scientific Technical: England, 1995. 